\title{
DCT Enabled Lifting Wavelet Transformation based Image Analysis for Brain Tumor Detection and Extraction
}

\author{
Nilesh B. Bahadure ${ }^{\# 1}$, Arun Kumar Ray ${ }^{* 2}$, Har Pal Thethi ${ }^{* 3}$ \\ ${ }^{\# 1}$ Research Scholar, School of Electronics Engineering, Kalinga Institute of Industrial Technology (KIIT) \\ University, Bhubaneswar, Odissa, India \\ ${ }^{2}$ School of Electronics Engineering, Kalinga Institute of Industrial Technology (KIIT) University, \\ Bhubaneswar, Odissa, India \\ ${ }^{3}$ Department of Electronics \& Telecommunication Engineering, Lovely Professional University (LPU), \\ Jalandhar, Punjab, India \\ ${ }^{1}$ nilesh_bahadure@rediffmail.com \\ ${ }^{3}$ harpalnitr@gmail.com
}

\begin{abstract}
This paper presents an accurate detection of a brain tumor by initial prediction of the tumor stage with the help of tumor infected magnetic resonance (MR) images. The accuracy of any brain tumor segmentation scheme depends on its ability to separately identify different classes of tissues. In fact, segmentation can be considered as the most important process in evaluating the characterization, delineation, and visualization of extracted portion i.e. region of interest from the MR images. This paper evaluates the ability of discrete cosine transformation and lifting wavelet transformation efficiently to segment different tissue classes and detect the tumor infected area through the MR images; thus, providing an improved technique which can help radiologists to accurately identify brain tumor grades, its exact location, size and its current stage. To the best of our knowledge and through the analysis, this is the first study of its kind that utilizes the performance of a discrete cosine transformation (DCT) enabled lifting wavelet transformation based clustering technique in detecting brain tumor. To summarize, our DCT enabled lifting wavelet transformation (LWT) based brain tumor detection algorithm abbreviated as DELWT (DCT Enabled Lifting Wavelet Transformation) provides promising efforts in brain tumor classification, detection, and extraction. It also has the potential for analysis and guiding methodology that are applicable in automatic analysis of larger data sets of MR images. The simulation results prove the significance and efficacy of the proposed mechanism in comparison to the existing techniques.
\end{abstract}

Keyword-Discrete cosine transformation (DCT), Histogram equalization, Lifting wavelet transformation (LWT), Morphology, Magnetic resonance imaging (MRI), Skull stripping

\section{INTRODUCTION}

Medical imaging is one of the most important, challenging and interesting areas as far as the detection and extraction of some important information from the magnetic resonance (MR) images or from computed tomography (CT) scan images is concerned [1]. Image segmentation is by far the most convincing and widely used techniques adopted for brain tumor detection from the MR images. In this study, discrete cosine transform enabled, lifting wavelet transformation based image segmentation algorithm is developed for the detection of brain tumor from the MR images and morphological operation in extracting the brain tumor from the MR images. Brain tumor segmentation process involves separating the different tumor tissues like edema, necrosis and solid tumor from the normal brain tissues, such as white matter, gray matter, and cerebrospinal fluid with the help of magnetic resonance images or other imaging modalities [2]-[6]. Unlike other imaging modalities, an MR image is a non-invasive and good soft tissue contrast imaging modality. It invariably provides valuable information about the shape, size, and the location of the brain tumors without exposing the patient to any kind of harmful radiation.

In this study, different MR sequence images are employed for diagnosis, including T1-weighted MR images, T2-weighted MR images, fluid-attenuated inversion recovery (FLAIR)-weighted MR images and proton density-weighted MR images. The detection of a brain tumor at an early stage is a key issue for providing improved treatment. Once a brain tumor is clinically suspected, radiological evaluation is required to determine its location, extent i.e. size and impact on the surrounding areas. On the basis of this information the best therapy, surgery, radiation or chemotherapy, is decided. It is evident that the chances of survival of a tumor infected patient can be increased significantly if the tumor is detected accurately in its early stage [15]. As a result, the study of brain tumors using imaging modalities has gained importance in the radiology department. 
The tumor is basically an uncontrolled growth of cancerous cells in any part of the body, whereas a brain tumor is an uncontrolled growth of cancerous cells in the brain. This uncontrolled growth of cells is classified as malignant (cancerous cells) or benign (non-cancerous cells). According to the World Health Organization, the most widely used grading scheme classifies a brain tumor into grade I to grade IV under the microscope. In general grade, I and II are the benign type of brain tumors, whereas grade III and IV are malignant types. The benign brain tumor is also called as low-grade brain tumor and if the low-grade brain tumor is left untreated, it is likely to develop into a high-grade brain tumor i.e. a malignant brain tumor [7], [8].

Although brain tumor can affect any part of the brain, it is mostly found in the posterior fossa in children and in the anterior two-thirds of the cerebral hemispheres in adults [9], [10]. Further, among adults, the most usual type of cancerous tumor is glial tumors, which has a high mortality rate. If a tumor is detected in a person of age 20 years or above, then over $90 \%$ of this tumor type is glial tumors [4]. The glial tumor occurs in the glial cells of the brain and spreads rapidly to healthy brain tissues.

Medical image segmentation for detection of brain tumor from the MR images or from other modern medical imaging modalities is a very important process for deciding right therapy at the right time. Madhukumar and Santhiyakumari [2] presented medical MR image segmentation technique based on fuzzy clustering means (FCM) and $\mathrm{K}$ - means of clustering and through experimental analysis proves, $\mathrm{K}$ - means is better than FCM, whereas, Abdel-Maksoud et al. [1] presented hybrid clustering technique, this combines the effect of K - means with FCM for medical image segmentation. This technique claims minimal computation time for segmentation and improved accuracy.

The extraction of the brain tumor area ejaculated from the processed image requires the segmentation of the brain MR images to two segments. One segment contains the tumor infected cells of the brain and the second contains the normal brain cells with information of gray matter, white matter, and the cerebral spinal fluid (CSF) [1]. Yang et al. [7] proposed a methodology for improved brain tumor clustering based on discrete wavelet transform (DWT). According to them, overall clustering accuracy is $94.8 \%$ and a balance error rate is $7.8 \%$. Kong et al. [3] investigated automatic segmentation of brain tissues from MR images using discriminative clustering and future selection approach. Demirhan et al. [4] proposed a new tissue segmentation algorithm using wavelets and neural networks, which segments brain MR images into the tumor, white matter, gray matter, edema, and cerebrospinal fluid. As proposed in the literature [11]-[13] presented a technique which employed texture features, and wavelet transformation algorithm for effective classification of dynamic contrast-enhanced MR images.

In recent years, with the advancement in healthcare systems, the emerging new imaging modalities, such as $\mathrm{X}$ - Rays, Ultrasonography, Magneto Encephalography (MEG), Electroencephalography (EEG), Positron Emission Tomography (PET), Computed Tomography (CT), Single Photon Emission Computed Tomography (SPECT) and Magnetic Resonance Imaging (MRI) [9], are used to show the detailed and almost complete information of a brain tumor [14], [16], thereby helping clinical experts to study the mechanism of the brain tumor for providing better treatment to the patients.

The proposed technique has an ability to segment brain tissues into healthy and tumor-infected tissues, and also fast and accurate when compared with the manual detection performed by radiologists or clinical experts. The experimental results conducted on the different MR images and comparison with the other state of the art techniques proves its significance.

\section{DeVelopment of Proposed Algorithm}

Flow diagram of the algorithm is shown in Fig. 1. The test images are magnetic resonance (MR) images of the brain. The magnetic resonance imaging (MRI) is always better than computed tomography (CT) scan images because there is no radiation in the magnetic resonance operation and also, the results obtained using MR images are more informative and clear as compared to CT scan images. These test images are then passed through the histogram equalization process, which is used to enhance the clarity of the images by removing the noises in the images. The magnetic resonance imaging contains a lot of noise, so, in this research, histogram equalization is used to smoothen the image quality. The algorithm to perform the segmentation and extraction of brain tumor from MR images is describes as follows: 


\section{START}

1. Input MR images

2. Preprocess to remove noises

3. Apply skull stripping

4. Set thresholding $(T) \geq 128$

If $\mathrm{T}<128$

(a) Find mask by assigning 1 to the white and 0 to the black pixels

(b) Multiply the mask with T1, T2 and FLAIR MR images

End

5. Calculate coefficients of DCT

6. Apply segmentation

7. Extract region of interest using morphology

STOP

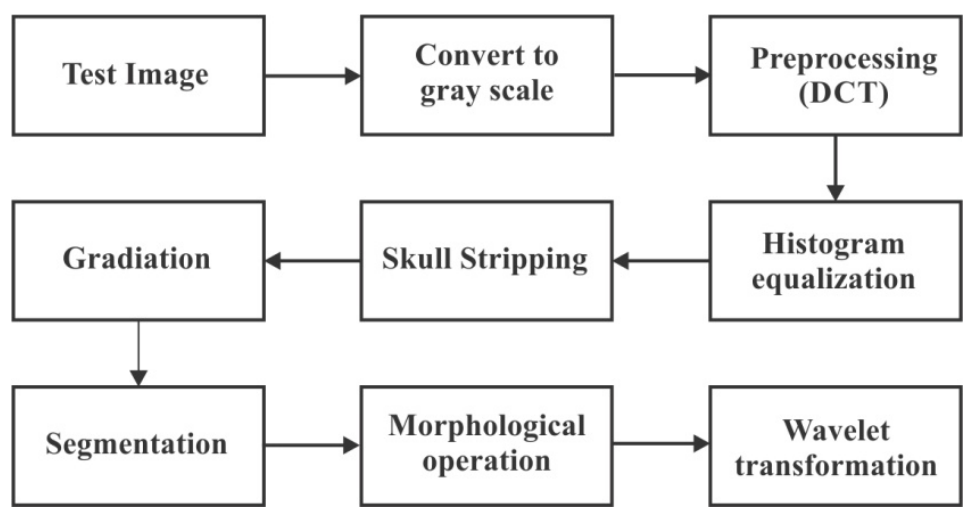

Fig. 1. Steps used in algorithm

\section{A. Pre-processing and Skull Stripping}

Preprocessing is used to normalize the intensity range of the MR images to $\left(\begin{array}{ll}0 & 1\end{array}\right)$ range by graduating all intensity values to the possible highest intensity value. Preprocessing also helps to improve the certain parameters of MR images like signal-to-noise ratio, smoothing the inner part of the region and preserving its edges [4].

For the proper analysis of the brain tumor from the MR images, the operation of skull stripping [17]-[19] is also required. Skull stripping is an important process in bio-medical image analysis and requires only in brain images for effective analysis [9]. It is not required in other medical image analysis such as of heart, lungs etc. Skull stripping is a process of eliminating all non-brain tissues from the brain images. With the help of skull stripping, it is possible to remove extra cerebral tissues such as skull, fat, and skin. There are various methods available for skull husking; some of the popular techniques are automatic skull stripping using image contour, skull stripping based on region growing and morphological operation and skull stripping based on histogram analysis or a threshold value. This study uses the skull husking technique that is based on a threshold operation to remove skull tissues.

\section{B. Gradiation and Morphological Operation}

Gradiation (gradient magnitude) is a process applied for the detection of the edges of the tumor infected area in the brain. This proposed methodology is designed in such a way that, this algorithm helps to determine the exact location of the tumor and the infected area in the brain.

The morphological operation is used for the extraction of the boundary areas of the brain images. Morphological image processing is a special area of image processing, which is used to process images based on shapes by collecting of non-linear operations related to shape or morphology of features in an image. If the morphological operation is processed on a binary image then it produces or creates a new binary image. The pixel has a non-zero value in a new binary image. 


\section{Segmentation}

Region growing is simple region-based image segmentation technique used for extracting an image region, which is interlinked with each other based on some predefined criteria [15]. These criteria usually depend on intensity information and/or edges in the image. In general, region growing requires a well-defined seed point which is chosen manually by an operator and extracts all pixels connected to the initial seed point, which is also based on some predefined criteria. One possible criterion based on edge detection might be used to grow the region until an edge in the image is met. This kind of segmentation scheme using region growing examines neighboring pixels of initial seed points and then finds the condition whether the pixel neighbors should be added to the region or not. The process is repetitively iterated, in the same manner as general data clustering algorithms.

The discrete cosine transform (DCT) helps to divide the image into sections of varied importance with respect to the images visual quality. The general equation for the one-dimensional DCT is defined as shown in Eq. (1) and the corresponding inverse one-dimensional DCT is shown in Eq. (2).

$$
\begin{gathered}
F(x)=\left(\frac{2}{N}\right)^{\frac{1}{2}} \sum_{i=0}^{N-1} \Lambda(i) \cos \left[\frac{\pi u}{2 N}(2 i+1)\right] f(i) \\
F^{-1}(x)=\Lambda(i)=\left\{\frac{1}{\sqrt{2}} \text { for } \varepsilon=0\right. \\
\Lambda(i)=1 \ldots \text { otherwise }
\end{gathered}
$$

The basic operation of the DCT consists of:

a) The input image is $\mathrm{N}$ by $\mathrm{M}$.

b) The pixel value $f(i, j)$ is the intensity of the pixel in $i^{\text {th }}$ row and $\mathrm{j}^{\text {th }}$ column.

c) $F(x, y)$ is an equivalent DCT coefficient in row $\mathrm{K} 1$ and column $\mathrm{K} 2$ of the DCT matrix.

d) In DCT most of its signal energy lies at lower frequencies, hence in most of the images, these may appear at the upper left corner of DCT.

e) With permissible distortion, it is possible to achieve the compression in the DCT, since the bottom right values in the DCT represents upper bound frequencies and are often too small, and hence can be neglected.

f) The DCT input is an 8 by 8 array of integers. This array contains gray scale values of each pixel.

g) Pixels in the size of 8 - bits have levels from 0 to 255 .

With the help of computers, it is easier to implement more efficient segmentation process, especially applicable to the field of medical and bio-medical imaging, where the higher contrast of the imaging is also an important factor. As DCT transformation is used to separate the images into different parts with the rejection of higher frequency components from the object, this algorithm is effectively suitable for the complicated imaging system.

\section{Lifting Wavelet Transformation}

A Wavelet is a function that is defined over a finite interval of time and has an average value of zero. The wavelet transformation technique is employed to develop functions, operators, data or information into components of different frequency, which enables studying each component separately. All the wavelets are generated or produced from the basic wavelet $\Psi(t)$ by scaling and translation process defined by the Eq. (3), basic wavelet is also referred as mother wavelet, because it is the point of origin for other wavelets.

$$
\Psi_{s, \tau}(t)=\frac{1}{\sqrt{s}} \Psi\left(\frac{t-\tau}{s}\right)
$$

Where $s$ and $\tau$ is the scale and translation factors, respectively. Lifting wavelet transformation [20] is a second generation wavelet transformation and using it all the classical wavelets can also be generated. Lifting is actually an algorithm through which wavelet transformation can be calculated efficiently [13]. The lifting scheme works entirely on the spatial domain and involves the following three steps for the transformation:

a) Split (Polyphase Decomposition)

b) Predict (P step) and

c) Update (U Step) 
These three transformation operations for one-dimensional lifting scheme using linear prediction on the signal $x$ are explained below:

(a) Splitting

Split the input signal $x$ into even and odd samples for even distribution of samples and it helps to process and transform the signals in reduced dimension.

\section{(b) Prediction}

Interpolation is used to predict the odd samples and it helps to balance the odd samples in contrast to even samples for processing and transformation.

\section{(c) Update}

To preserve the mean value of the samples, even samples must be updated at regular intervals.

The generalized lifting stage of wavelet decomposition and its equivalent inverse wavelet synthesis is shown in Fig. 2.

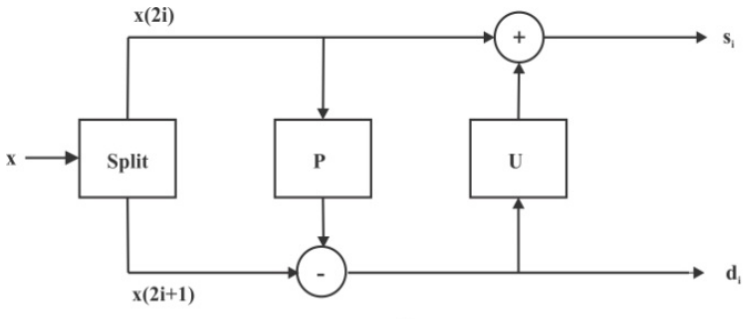

(a)

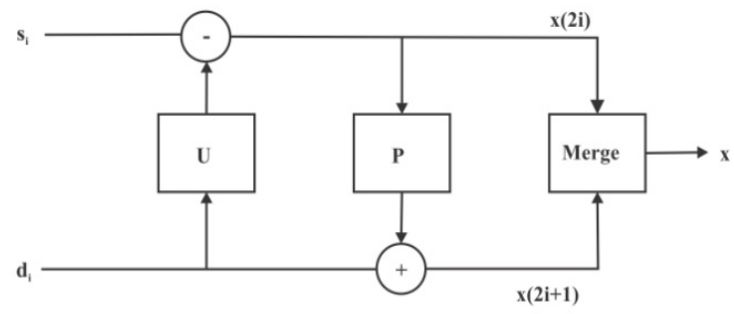

(b)

Fig. 2. Generalized lifting stages: (a) wavelet decomposition, (b) inverse wavelet

\section{III.RESULTS AND DISCUSSIONS}

This section presents the results of our proposed image segmentation technique, which are obtained by brain MR images. The dataset used for the experimentation and results obtained are given in the next subsections

\section{A. Dataset}

This section presents the materials, the source of brain MR image datasets and the algorithm to perform brain MR tissue segmentation. The test images are magnetic resonance (MR) images of the brain, including T1weighted MR images with TR/TE: 1740/20, T2-weighted MR images with TR/TE: 5850/130, and FLAIRweighted MR images with TR/TE: 8500/130. These test images were acquired using a 1.5 Tesla General Electric MR machine. The proposed methodology is applied to real datasets including brain MR images of 512 $\times 512$ pixel size. For the purpose of the analysis and to validate the results of proposed method, we also considered 22 images from the Digital Imaging and Communications in Medicine (DICOM) dataset [24], all of which included are tumor infected brain tissues and 44 images from Brain Web dataset [25].

\section{B. Performance Matrices}

To validate the performance of the proposed algorithm, the performance matrices such as mean, standard deviation, mean square error (MSE), peak signal-to-noise ratio (PSNR), median, bit error rate, correlation factor, variance are calculated. The formulas for some of the useful parameters are shown below:

(1) Mean (M):

$$
\text { Mean }=\left(\frac{1}{n \times m}\right) \sum_{0}^{n-1} \sum_{0}^{m-1} f(n, m)
$$

Where $\mathrm{n}$ and $\mathrm{m}$ are image size. Lower value indicates good amount of noise elimination from in image.

(2) Standard Deviation (SD)

$$
S D=\sqrt{\left(\frac{1}{n \times m}\right)\left(\sum_{0}^{n-1} \sum_{0}^{m-1}(f(n, m)-\mu)^{2}\right)}
$$

Higher value indicates better intensity level and high contrast of edges of an image.

(3) Mean Square Error (MSE)

$$
M S E=\left(\frac{1}{M \times N}\right)\left(\sum \sum\left(I(i, j)-I^{R}(i, j)\right)^{2}\right.
$$

where $I(i, j)$ and $I^{R}(i, j)$ indicates original and reconstructed image respectively 
(4) Peak Signal to Noise Ratio (PSNR)

$$
\text { PSNR in } d B=20 \log _{10}\left(\frac{2^{n}-1}{M S E}\right)
$$

Lower value of MSE and higher value of PSNR indicates better signal to noise ratio

\section{Experimental Results}

The proposed algorithm was carried out using Matlab 7.12.0 (R2011a), which runs on Windows 8 operating system, and has an Intel core i3 processor and a 4GB RAM. Fig. 3 shows the preprocessing results for the region of growing effect and Fig. 4 shows the results for the different stages of the operation for the detection of brain tumor and extraction of a region of interest. The cross distance parametric analysis of the extracted area of the brain tumor is shown in Fig. 5. Different performance parameters like mean, standard deviation (SD), median (Med), bit error rate (BER), correlation factor (C), variance (Var), mean squared error (MSE), and peak signal to noise ratio (PSNR) for the tumor detection and for the region of interest is shown in Table I and Table II respectively. For better visualization, lower value of MSE and higher value of PSNR is recommended. Similarly, when the low value is obtained for mean and higher value for the standard deviation (SD) then it indicates high contrast and high intensity respectively. The area of the extracted brain tumor is represented in square cms and a pixel with comparison with the area calculated by the expert radiologist is shown in Table III. The DCT-LWT (DELWT) based techniques give better performance and are more informative as compared to the other available state of the art techniques that are based on watershed segmentation and soft computing [21][23].

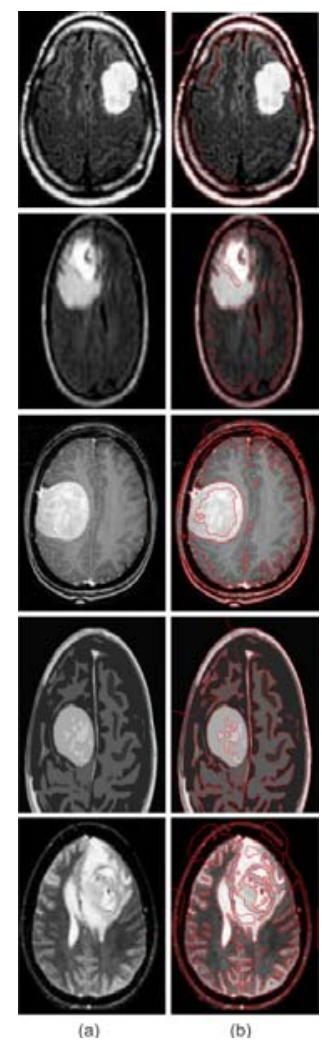

Fig. 3. Result of pre-processing: (a) original image, (b) region of growing. 


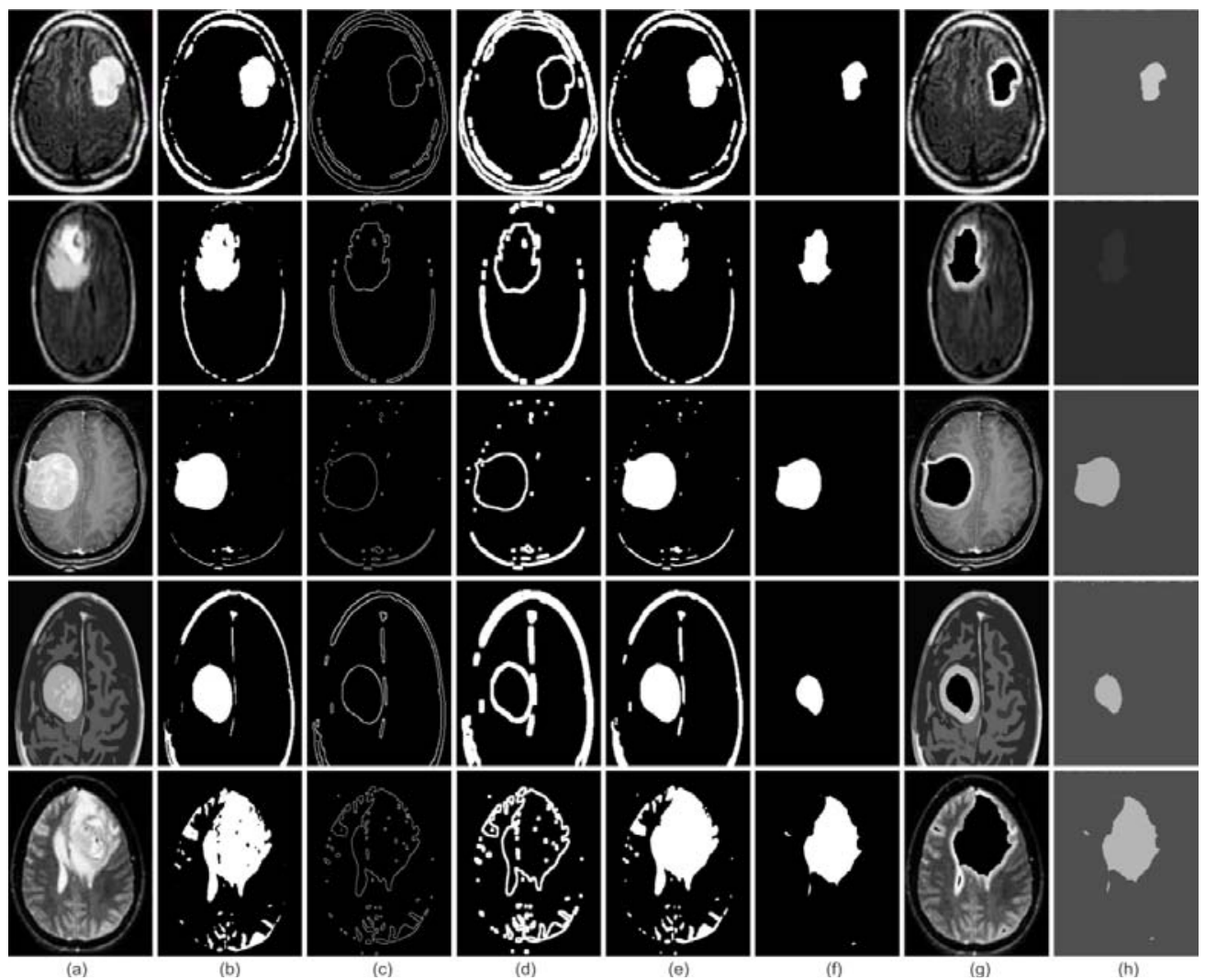

Fig. 4. Result of brain detection and extraction: (a) original image (b) cluster image (c) binary gradient image (d) dilated gradient image (e) intense segmented image $(\mathrm{f})$ erosion image $(\mathrm{g})$ subtracted image $(\mathrm{h})$ distortion-less restored image.

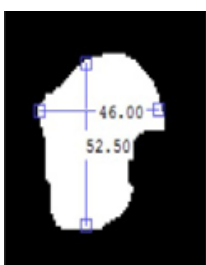

(a)

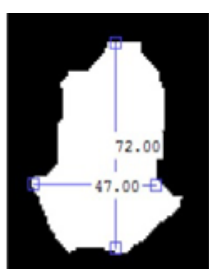

(b)

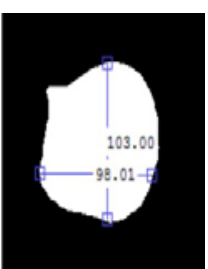

(c)

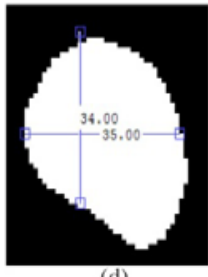

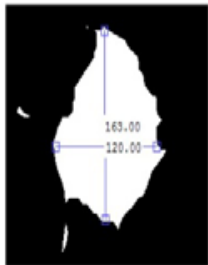

(e)

Fig.5. Cross-sectional distance of extracted tumor from brain MR image.

TABLE I. Performance Analysis Parameters for Segmented Tissues

\begin{tabular}{|c|c|c|c|c|c|c|c|c|c|c|}
\hline Image & Size & $\begin{array}{c}\text { No. of } \\
\text { iteration }\end{array}$ & $\begin{array}{c}\text { No. of } \\
\text { errors }\end{array}$ & BER & Correlation & Mean & SD & Var & Med & $\begin{array}{c}\text { Elapsed } \\
\text { time (in sec) }\end{array}$ \\
\hline $\operatorname{Im} 1$ & $\begin{array}{c}274 x \\
278\end{array}$ & 5040 & 18 & 0.017 & 0.9640 & 65.61 & 69.34 & 4808 & 47 & 1.0149 \\
\hline $\operatorname{Im} 2$ & $\begin{array}{c}257 \mathrm{x} \\
256\end{array}$ & 720 & 27 & 0.026 & 0.9460 & 43.37 & 57.74 & 3335 & 30 & 1.0128 \\
\hline $\operatorname{Im} 3$ & $\begin{array}{c}336 \mathrm{x} \\
407\end{array}$ & 1430 & 30 & 0.029 & 0.9397 & 75.49 & 69.34 & 4808 & 79 & 0.9860 \\
\hline $\operatorname{Im} 4$ & $\begin{array}{c}200 \mathrm{x} \\
198\end{array}$ & 1650 & 39 & 0.038 & 0.9231 & 60.30 & 52.48 & 2754 & 43 & 1.0124 \\
\hline $\operatorname{Im} 5$ & $\begin{array}{c}336 \mathrm{x} \\
432\end{array}$ & 1400 & 28 & 0.027 & 0.9440 & 68.00 & 72.77 & 5295 & 50 & 1.0034 \\
\hline
\end{tabular}


TABLE II. Performance Analysis Parameters for Region of Interest

\begin{tabular}{|c|c|c|c|c|c|c|c|}
\hline Image & Size & Mean & SD & Var & Med & MSE & PSNR \\
\hline $\operatorname{Im} 1$ & $274 \times 278$ & 0.3971 & 164.88 & 27186 & 0 & 1.92 & $45.34 \mathrm{~dB}$ \\
\hline $\operatorname{Im} 2$ & $257 \times 256$ & 0.0602 & 125.02 & 15632 & 0 & 0.31 & $53.25 \mathrm{~dB}$ \\
\hline $\operatorname{Im} 3$ & $336 \times 407$ & 0.9668 & 176.31 & 31087 & 0 & 4.43 & $41.69 \mathrm{~dB}$ \\
\hline $\operatorname{Im} 4$ & $200 \times 198$ & 0.4507 & 137.88 & 19013 & 0 & 2.10 & $44.94 \mathrm{~dB}$ \\
\hline $\operatorname{Im} 5$ & $336 \times 432$ & 1.4853 & 170.76 & 29161 & 0 & 6.61 & $39.96 \mathrm{~dB}$ \\
\hline
\end{tabular}

TABLE III. Area of the Extracted Tumor from MR Images

\begin{tabular}{|c|c|c|c|c|c|}
\hline Image & Size & $\begin{array}{c}\text { Area in } \\
\text { pixel }\end{array}$ & $\begin{array}{c}\text { Area in } \\
\mathbf{c m}^{\mathbf{2}}\end{array}$ & $\begin{array}{c}\text { Area } \\
\text { ratio }\end{array}$ & $\begin{array}{c}\text { Accuracy of the area in comparison with } \\
\text { the area calculated by expert radiologist }\end{array}$ \\
\hline $\operatorname{Im} 1$ & $274 \times 278$ & 76172 & 1.22 & 0.0002231 & $98.2 \%$ \\
\hline $\operatorname{Im} 2$ & $257 \times 256$ & 65792 & 0.58 & 0.0000282 & $100 \%$ \\
\hline $\operatorname{Im} 3$ & $336 \times 407$ & 136752 & 1.45 & 0.0000731 & $99.7 \%$ \\
\hline $\operatorname{Im} 4$ & $200 \times 198$ & 39600 & 0.23 & 0.0000008 & $98.5 \%$ \\
\hline $\operatorname{Im} 5$ & $336 \times 432$ & 145152 & 2.79 & 0.0002342 & $100 \%$ \\
\hline
\end{tabular}

\section{CONCLUSION}

In this study, using the MR images of the brain, we segmented brain tissues into healthy and tumor-infected tissues. Fifteen patients infected with a glial tumor, all in malignant stages, assisted in this study. We used preprocessing to improve the signal-to-noise ratio. We used skull stripping with gradient magnitude to improve the skull stripping performance. Further, we used the discrete cosine transformation and wavelet technique to segment the images and for future extraction. From the experimental results performed on the different images, it is cleared that the analysis for the brain tumor detection is fast and accurate when compared with the manual detection performed by radiologists or clinical experts. The various performance analysis factors also indicate that the DCT-LWT based algorithm provides better result by improving certain parameters such as mean, standard deviation, MSE, and PSNR [21]-[23]. Our experimental results show that the DCT-LWT based approach can aid in the accurate and timely detection of brain tumor along with the identification of its exact location. To conclude, our DCT enabled LWT based brain tumor detection algorithm provides improvised techniques for brain tumor classification, detection, and extraction. It also has a potential for analysis and guiding methodology which are applicable to the automatic analysis of larger data sets of MR images.

\section{ACKNOWLEDGMENT}

The research was supported by the Chhattisgarh Council of Science \& Technology Raipur - Ministry of Science \& Technology, Chhattisgarh State, India (grant No. 1928/CCOST/2015).

\section{REFERENCES}

[1] E. Abdel-Maksoud, M. Elmogy and R. Al-Awadi, "Brain tumor segmentation based on a hybrid clustering technique," Egypt Inform J, vol. 16, pp. 71-81, 2015.

[2] S. Madhukumar and N. Santhiyakumari, "Evaluation of k-means and fuzzy c-means segmentation on MR images of brain," The Egypt J Radiol Nucl Med, vol. 46, pp. 475-479, 2015.

[3] Y. Kong, Y. Deng and Q. Dai, "Discriminative clustering and feature selection for brain MRI segmentation," IEEE Signal Proc Let, vol. 22, pp. 573-577, 2015.

[4] A. Demirhan, M. Toru and I. Guler, "Segmentation of tumor and edema along with healthy tissues of brain using wavelets and neural networks," IEEE J Biomed Health Inform, vol. 19, pp. 1451-1458, 2015.

[5] M.T. El-Melegy and H.M. Mokhtar, "Tumor segmentation in brain MRI using a fuzzy approach with class center priors," EURASIP J Image Video Process, vol. 21, pp. 1-14, 2014.

[6] N. Gordillo, M. Eduard and S. Pilar, "State of the art survey on MRI brain tumor segmentation," Magn Reson Imaging, vol. 31, pp. $1426-1438,2013$.

[7] G. Yang, T. Nawaz, T.R. Barrick, F.A. Howe and G. Slabaugh, "Discrete wavelet transform-based whole-spectral and subspectral analysis for improved brain tumor clustering using single voxel MR spectroscopy," IEEE T Biomed Eng, vol. 62, pp. 2860-2866, 2015.

[8] K.S. Tamilselvan, G. Murugesan and B. Gnanasekaran, "Brain tumor detection from clinical CT and MRI images using wt-fcm algorithm," IEEE International Conference on Green Computing, Communication and Conservation of Energy (ICGCE) 12-14 December 2013, Chennai, Tamilnadu, India, pp. 260-263.

[9] C.C. Benson and V.L. Lajish, "Morphology based enhancement and skull stripping of MRI brain images," in Proc. of IEEE International Conference on Intelligent Computing Applications, 6-7 March 2014,Coimbatore, Tamilnadu, India, pp. 254-257.

[10] H.A. Aslam, T. Ramashri and M.I.A. Ahsan, "A new approach to image segmentation for brain tumor detection using pillar k-means algorithm,” Int J Adv Res Comput Commun Eng, vol. 2, pp. 1429-1436, 2013.

[11] T. Torheim, E. Malinen, K. Kvaal, H. Lyng, U.G. Indahl, E.K.F. Andersen and C.M. Futsaether, "Classification of dynamic contrast enhanced MR images of cervical cancers using texture analysis and support vector machines," IEEE Trans Med Imag, vol. 33, pp. $1648-1656,2014$.

[12] L. Guo, L. Zhao, Y. Wu, Y. Li, G. Xu and Q. Yan, "Tumour detection in MR images using one-class immune feature weighted SVMs," IEEE Trans Magn, vol. 47, pp. 3849-3852, 2011. 
[13] J. Yao, J. Chen and C. Chow, "Breast tumour analysis in dynamic contrast enhanced MRI using texture features and wavelet transform," IEEE J Sel Topics Signal Process, vol. 3, pp. 94-100, 2009.

[14] J. Liu J, M. Li, J.Wang, F.Wu, T. Liu and Y. Pan, "A survey of MRI-based brain tumor segmentation methods," Tsinghua Sci Technol, vol. 19, pp. 578-595, 2014.

[15] G. Coatrieux, H. Huang, H. Shu, L. Luo and C. Roux, "A watermarking based medical image integrity control system and an image moment signature for tampering characterization," IEEE J Biomed Health Inform, vol. 17, pp. 1057-1067, 2013.

[16] R.C. Gonzalez and R.E. Woods, Digital Image Processing, 2nd ed. Upper Saddle River, New Jersey, USA, Prentice Hall 2002.

[17] S.Z. Oo and A.S. Khaing, "Brain tumor detection and segmentation using watershed segmentation and morphological operation," Int J Res Eng Technol, vol. 3, pp. 367-374, 2014.

[18] R. Roslan, N. Jamil and R. Mahmud, "Skull stripping magnetic resonance images brain images: region growing versus mathematical morphology," Int J Comput Inf Syst Ind Manage Appl, vol. 3, pp. 150-158, 2011.

[19] S. Mohsin, S. Sajjad, Z. Malik and A.H. Abdullah, "Efficient way of skull stripping in MRI to detect brain tumor by applying morphological operations, after detection of false background," Int J Inf Edu Technol, vol. 2, pp. 335-337, 2012

[20] T. Acharya and C. Chakrabarti, "A survey on lifting-based discrete wavelet transform architectures," J VLSI Signal Proc, vol. 42, pp. 321-339, 2006

[21] J. Yang, J. Fan, D. Ai, S. Zhou, S. Tang and Y. Wang, "Brain MR image denoising for Rician noise using pre-smooth non-local means filter," Biomed Eng Online, vol. 14, pp. 1-20, 2015.

[22] S. Mohane and M. Borse, "Comparitive study of brain tumor detection using morphological operators," Int J Res Eng Technol, vol. 4, pp. $422-428,2015$.

[23] D. Anithadevi and K. Perumal, "Brain tumour extraction based on segmentation,” Int J Recent Innov Trend Comput Commun, vol. 2 , pp. 2682-2689, 2014.

[24] DICOM Samples Image Sets. [Online]. Available: http://www.osirixviewer.com/datasets/

[25] Brain web: Simulated Brain Database. [Online]. Available: http://brainweb.bic.mni.mcgill.ca/cgi/brainweb1

\section{AUTHOR PROFILE}

Nilesh B. Bahadure (corresponding author) was born in 1978. He received the Bachelor of Engineering degree from Department of Electronics Engineering, Nagpur University, in 2000 and received Master of Engineering in Digital Electronics from Amravati University, in 2005. He is now a Ph.D. Scholar in the School of Electronics, Kalinga Institute of Industrial Technology (KIIT) University, Bhubaneswar since 2013. His research interests include Computer Vision \& Image Processing, Signal Processing, Embedded Systems and Medical Electronics. He had published one book on Advanced Microprocessor published by PHI Learning Pvt. Limited, and was reviewed one book on Basic Electrical Engineering, Published by Pearson Education Pvt. Limited. He had 9 publications in International and National Journals and 11 papers presented in International and National conferences. He is a Life Member of various institutions like ISTE, IETE, IE (India), SESI, ISRS, ISCA and IAENG. (Email: nbahadure@gmail.com)

Dr. Arun Kumar Ray is currently Professor \& Dean School of Electronics at KIIT University Bhubaneswar, Odissa. His research interest includes Computer Vision and Image Processing, Biomedical Engineering, Fuzzy Logic and Neural Network, and Digital Signal Processing. He is a Life member of various institutions like ISTE, IETE, IE (India) and active member of international professional bodies like IEEE, and Institution of Engineering and Technology (IET). He is also serving as a Member in the Editorial board of some reputed international journals.

Dr. Har Pal Thethi is currently Associate Director and Professor at Lovely Professional University, Jalander, Punjab, India. He received his B.Tech from BIET Bhadrak, M.Tech from NIT Rourkela and PhD in Electronics Engineering from KIIT University Bhubaneswar. His research interest includes Computer Vision and Image Processing, Pattern Recognition, Soft Computing, and Digital Signal Processing. He is a Life Member of various institutions like ISTE, ISCA. He is also an active member of international professional bodies like IEEE, Institution of Engineering and Technology (IET). 